EUROPEAN JOURNAL OF PURE AND APPLIED MATHEMATICS

Vol. 12, No. 1, 2019, 208-225

ISSN 1307-5543 - www.ejpam.com

Published by New York Business Global

\title{
An application of le-semigroup techniques to semigroups, $\Gamma$-semigroups and to hypersemigroups
}

Niovi Kehayopulu

\begin{abstract}
An le-semigroup, is a semigroup $S$ at the same time a lattice with a greatest element $e(e \geq a$ for every $a \in S)$ such that $a(b \vee c)=a b \vee a c$ and $(a \vee b) c=a c \vee b c$ for all $a, b, c \in S$. If $S$ is not a lattice but only an upper semilattice ( $\vee$-semilattice), then is called $\vee e$-semigroup. A poe-semigroup is a semigroup $S$ at the same time an ordered set with a greatest element $e$ such that $a \leq b$ implies $a c \leq b c$ and $c a \leq c b$ for all $c \in S$. Every $\vee e$-semigroup is a poe-semigroup. If $S$ is a semigroup or a $\Gamma$-semigroup, then the set $\mathcal{P}(S)$ of all subsets of $S$ is an le-semigroup. If $S$ is an hypersemigroup, then the set $\mathcal{P}^{*}(S)$ of all nonempty subsets of $S$ is an le-semigroup. So all the results of le-semigroups, $\vee e$-semigroups and poe-semigroups based on ideal elements, automatically hold for semigroups, $\Gamma$-semigroups and hypersemigroups. This is not the case for ordered $\Gamma$-semigroups or ordered hypersemigroups; however the main idea, even in these cases, comes from the le ( $\vee$ e)-semigroups. As an example, we study the weakly prime ideal elements of a $\vee e$-semigroup and their role to the different type of semigroups mentioned above.
\end{abstract}

2010 Mathematics Subject Classifications: 06F05, 06F99, 20M99, 20M10

Key Words and Phrases: Lattice ordered semigroup, le-semigroup, (ordered) $\Gamma$-semigroup, (ordered) hypersemigroup, weakly prime, weakly semiprime, prime subset, right (left) ideal element, right (left) ideal

\section{Introduction}

If $S$ is a semigroup or a $\Gamma$-semigroup, then the set of (all) subsets of $S$ is a poesemigroup, a $\vee e$-semigroup and an le-semigroup. If $S$ is an hypersemigroup, then the set of (all) nonempty subsets of $S$ is a poe-semigroup, a $\vee e$-semigroup and an le-semigroup. Every $l e$-semigroup is a $V e$-semigroup and every $V e$-semigroup is a poe-semigroup. So many results on $\Gamma$-semigroups or hypersemigroups do not need any proof, unless we would like to know how an independent proof on these structures works. This is not the case for ordered semigroups, ordered $\Gamma$-semigroups or ordered hypersemigroups; however the main idea, even in these cases, comes from the le $(\vee e)$ or poe-semigroups.

In the present paper we first characterize the weakly prime and weakly semiprime ideal elements of a $\vee e$-semigroup $S$ in terms of right and left ideal elements of $S$ and show that

DOI: https://doi.org/10.29020/nybg.ejpam.v12i1.3383

Email address: nkehayop@math.uoa.gr (N. Kehayopulu) 
one can get the corresponding characterizations of weakly prime and weakly semiprime ideals of semigroups, $\Gamma$-semigroups or hypersemigroups (in terms of right and left ideals), as corollaries. Then we examine the same results in case of an ordered semigroup, an ordered $\Gamma$-semigroup and an ordered hypersemigroup.

For weakly prime ideals of rings see, for example, [5].

This is from the first chapter "What can lattices do for you?" by Garrett Birkhoff in [Trends in Lattice Theory. Contributors: Garrett Birkhoff, Samuel S. Holland, JR., Henry Crapo and Gian-Carlo Rota, George Grätzer. Van Nostrand Reinhold Comp. 1970]: "In general, lattice theory has helped to simplify, unify and generalize many aspects of mathematics ..." (p. 1); "Because of its central concept, that of order, intertwines through almost all of mathematics ..." (p. 1); “... lattices can do things for you no matter what kind of mathematician you are!" (p. 38).

\section{Preliminaries}

An ordered groupoid (po-groupoid) is a groupoid $S$ at the same time an ordered set such that $a \leq b$ implies $a c \leq b c$ and $c a \leq c b$ for all $c \in S$. A $\vee$-groupoid is a groupoid $S$ at the same time an upper semilattice such that $(a \vee b) c=a c \vee b c$ and $a(b \vee c)=a b \vee a c$ for all $a, b, c \in S$. If $S$ is not only an upper semilattice, but a lattice, then it is called a lattice ordered semigroup (or an l-semigroup) [2,4] (see also [1]). By a poe-groupoid, Ve-groupoid or le-groupoid we mean a po-groupoid, $\vee$-groupoid or $l$-groupoid $S$, respectively, having a greatest element usually denoted by " $e$ " (i.e. $e \geq a$ for every $a \in S$ ). If the multiplication on a po-groupoid $S$ is associative, then $S$ is called a po-semigroup. In a similar way we have the Ve-semigroups and the le-semigroups.

In a poe-groupoid $S$, an element $a$ is called a right ideal element if $a e \leq a$, it is called a left ideal element if $e a \leq a$. An element which is both a right and a left ideal element is called an ideal element [6] (see also [2; p. 328]). We denote by $F_{r}\left(\right.$ resp. $F_{l}$ ) the set of right (resp. left) ideal elements of $S$.

A nonempty set $A$ of a groupoid $(S, \cdot)$ is called a right (resp. left) ideal of $S$ if $A S \subseteq A$ (resp. $S A \subseteq A$ ). It is called an ideal of $S$ if it is both a right and left ideal of $S[3,17]$. If $S$ is an ordered groupoid, then a nonempty subset $A$ of $S$ is called a right (resp. left) ideal of $S$, if it is an ideal of the semigroup $(S, \cdot)$ and, in addition, if $a \in A$ and $S \ni b \leq a$, then $b \in A[7]$. Either for a groupoid or for an ordered groupoid, sets that are both right and left ideals are called ideals.

For sets $A, B$ and $\Gamma$, the symbol $A \Gamma B$ denotes the set of all $a \gamma b$ with $a \in A, b \in B$, $\gamma \in \Gamma$. If $A=\emptyset$ or $B=\emptyset$, we define $A \Gamma B=\emptyset$.

Definition 2.1. [10] Let $S$ and $\Gamma$ be two nonempty sets. The set $S$ is called a $\Gamma$-groupoid if the following two assertions are satisfied:

(1) $S \Gamma S \subseteq S$.

(2) If $a, b, c, d \in S$ and $\gamma, \mu \in \Gamma$ such that $a=c, b=d$ and $\gamma=\mu$, then $a \gamma b=c \mu d$.

If, in addition, for all $a, b, c \in S$ and all $\gamma, \mu \in \Gamma$, we have the property 
(3) $(a \gamma b) \mu c=a \gamma(b \mu c)$

then $S$ is called a $\Gamma$-semigroup.

Definition 2.1 is the definition of a $\Gamma$-semigroup introduced by Sen and Saha in [19] in which the missing uniqueness condition (2) has been added (for details, see [10]).

In other words, a $\Gamma$-semigroup is a set of binary operations on $S$ and condition (3) of Definition 2.1 is satisfied.

For an application of $\Gamma$-semigroup techniques to the Green's theorem we refer to [14].

Let us give an example of a $\Gamma$-semigroup (see [10; Example 2(B)]): Consider the set $S=\{a, b, c\}$ and let $\Gamma=\{\gamma, \mu\}$ be the set of two binary operations on $S$ defined by the tables below:

\begin{tabular}{c|c|c|c}
$\gamma$ & $a$ & $b$ & $c$ \\
\hline$a$ & $a$ & $b$ & $c$ \\
\hline$b$ & $b$ & $c$ & $a$ \\
\hline$c$ & $c$ & $a$ & $b$
\end{tabular}

\begin{tabular}{c|c|c|c}
$\mu$ & $a$ & $b$ & $c$ \\
\hline$a$ & $b$ & $c$ & $a$ \\
\hline$b$ & $c$ & $a$ & $b$ \\
\hline$c$ & $a$ & $b$ & $c$
\end{tabular}

We have $(x \rho y) \omega z=x \rho(y \omega z)$ for all $x, y, z \in S$ and all $\rho, \omega \in \Gamma$, so $S$ is a $\Gamma$-semigroup.

A $\Gamma$-groupoid $(S, \Gamma)$ endowed with an order relation " $\leq$ " such that $a \leq b$ implies $a \gamma c \leq b \gamma c$ and $c \gamma a \leq c \gamma b$ for all $c \in S$ is called an ordered $\Gamma$-groupoid (po-Г-groupoid) [20].

A nonempty subset $A$ of a $\Gamma$-groupoid $S$ is called a right (resp. left) ideal of $S$ if $A \Gamma S \subseteq A$ (resp. $S \Gamma A \subseteq A$ ). A nonempty subset $A$ of a po- $\Gamma$-groupoid $S$ is called a right (resp. left) ideal of $S$ if it is a right (resp. left) ideal of the $\Gamma$-semigroup $S$ and, in addition, if $a \in A$ and $S \ni b \leq a$, then $b \in A$ [10]. In both cases, $A$ is called an ideal of $S$ if it is both a right and a left ideal of $S$.

Remark 2.2. If $(S, \Gamma)$ is a $\Gamma$-semigroup then, for any subsets $A, B, C$ of $S$, we have $(A \Gamma B) \Gamma C=A \Gamma(B \Gamma C)$. Indeed, if $x \in(A \Gamma B) \Gamma C$, then $x=(a \gamma b) \mu c=a \gamma(b \mu c)$ for some $a, b, c \in S$ and $\gamma, \mu \in \Gamma$ and so $x \in A \Gamma(B \Gamma C)$. Similarly we have $A \Gamma(B \Gamma C) \subseteq(A \Gamma B) \Gamma C$.

Lemma 2.3. If $(S, \Gamma)$ is a $\Gamma$-groupoid then, for any subsets $A, B, C$ of $S$, we have

(1) $(A \cup B) \Gamma C=A \Gamma C \cup B \Gamma C$ and

(2) $A \Gamma(B \cup C)=A \Gamma B \cup A \Gamma C$.

Proof. (1) Since $A \cup B \supseteq A$ and $A \cup B \supseteq B$, we have $(A \cup B) \Gamma C \supseteq A \Gamma C$ and $(A \cup B) \Gamma C \supseteq$ $B \Gamma C$ and so $(A \cup B) \Gamma C \supseteq A \Gamma C \cup B \Gamma C$. Let now $x \in(A \cup B) \Gamma C$. Then $x=t \gamma c$ for some $t \in A \cup B, \gamma \in \Gamma, c \in C$. If $t \in A$, then $x \in A \Gamma C$; if $t \in B$, then $x \in B \Gamma C$ and so $x \in A \Gamma C \cup B \Gamma C$. The proof of property (2) is similar.

An hypergroupoid is a nonempty set $S$ with an hyperoperation

$\circ: S \times S \rightarrow \mathcal{P}^{*}(S) \mid(a, b) \rightarrow a \circ b$ on $S$

and an operation $*: \mathcal{P}^{*}(S) \times \mathcal{P}^{*}(S) \rightarrow \mathcal{P}^{*}(S) \mid(A, B) \rightarrow A * B$ on $\mathcal{P}^{*}(S)$ (induced by the operation of $S)$ such that $A * B=\bigcup_{(a, b) \in A \times B}(a \circ b)$ for every $A, B \in \mathcal{P}^{*}(S)$. $\mathcal{P}^{*}(S)$ denotes the set of all nonempty subsets of $S$. Clearly, $x \in A * B$ if and only if there exist 
$a \in A$ and $b \in B$ such that $x \in a \circ b$. As one can easily see, for any $x, y \in S$, we have $\{x\} *\{y\}=x \circ y$. An hypergroupoid $S$ is called hypersemigroup if $\{x\} *(y \circ z)=(x \circ y) *\{z\}$ for every $x, y, z \in S[11,13]$.

The concept of an ordered groupoid can be naturally transferred to ordered hypergroupoids as follows: An hypergroupoid $(S, \circ)$ is called ordered hypergroupoid if there exists an order relation " $\leq$ " on $S$ such that $a \leq b$ implies $a \circ c \preceq b \circ c$ and $c \circ a \preceq c \circ b$ for any $c \in S$ in the sense that for every $u \in a \circ c$ there exists $v \in b \circ c$ such that $u \leq v$ and for every $u \in c \circ a$ there exists $v \in c \circ b$ such that $u \leq v$ [18].

A nonempty subset $A$ of an hypergroupoid $(S, \circ)$ is called a right (resp. left) ideal of $S$ if $A * S \subseteq A$ (resp. $S * A \subseteq A$ ). If, in particular, the hypergroupoid $S$ is an ordered hypergroupoid, then a set $A$ is called a right (resp. left) ideal of $S$ if $A$ is a right (resp. left) ideal of the hypergroupoid $(S, \circ)$ and, in addition if $a \in A$ and $S \ni b \leq a$ implies $b \in A$. Both for hypergroupoids or ordered hypergroupoids the sets that are at the same time right and left ideals are called ideals.

Lemma 2.4. [13; Proposition 9] For an hypersemigroup $(S, \circ)$ and nonempty subsets $A, B, C$ of $S$, we have

$$
A *(B * C)=(A * B) * C
$$

The following lemma has been proved in a more general case in [13; Proposition 7]. Since this lemma plays an essential role in the present paper, for the sake of completeness, we will give its proof.

Lemma 2.5. If $(S, \circ)$ is an hypergroupoid then, for any nonempty subsets $A, B, C$ of $S$, we have

(1) $(A \cup B) * C=(A * C) \cup(B * C)$ and

(2) $A *(B \cup C)=(A * B) \cup(A * C)$.

Proof. Since $A \cup B \supseteq A, B$, we have $(A \cup B) * C \supseteq(A * C) \cup(B * C)$. If $x \in(A \cup B) * C$, then $x \in u \circ c$ for some $u \in A \cup B, c \in C$. If $u \in A$, then $x \in A * C$; if $u \in B$, then $x \in B * C$, so $x \in(A * C) \cup(B * C)$.

Definition 2.6. [7, 8] Let $S$ be a groupoid or an ordered groupoid. A subset $M$ of $S$ is called prime if for any subsets $A, B$ of $S$ such that $A B \subseteq M$, we have $A \subseteq M$ or $B \subseteq M$. It is called semiprime if for any subset $A$ of $S$ such that $A^{2} \subseteq M$, we have $A \subseteq M$.

Definition 2.7. [7,8] Let $S$ be a groupoid or an ordered groupoid. A subset $M$ of $S$ is called weakly prime if for any ideals $A, B$ of $S$ such that $A B \subseteq M$, we have $A \subseteq M$ or $B \subseteq M$. It is called weakly semiprime if for any ideal $A$ of $S$ such that $A^{2} \subseteq M$, we have $A \subseteq M$.

Definition 2.8. [9] Let $S$ be a $\Gamma$-groupoid or an ordered $\Gamma$-groupoid. A subset $M$ of $S$ is called prime if for any subsets $A, B$ of $S$ such that $A \Gamma B \subseteq M$, we have $A \subseteq M$ or $B \subseteq M$. It is called semiprime if for any subset $A$ of $S$ such that $A \Gamma A \subseteq M$, we have $A \subseteq M$. 
Definition 2.9. [9] Let $S$ be a $\Gamma$-groupoid or an ordered $\Gamma$-groupoid. A subset $M$ of $S$ is called weakly prime if for any ideals $A, B$ of $S$ such that $A \Gamma B \subseteq M$, we have $A \subseteq M$ or $B \subseteq M$. It is called weakly semiprime if for any ideal $A$ of $S$ such that $A \Gamma A \subseteq M$, we have $A \subseteq M$.

Definition 2.10. [15] Let $(S, \circ)$ be an hypergroupoid or an ordered hypergroupoid. A subset $M$ of $S$ is called prime if for any nonempty subsets $A, B$ of $S$ such that $A * B \subseteq M$, we have $A \subseteq M$ or $B \subseteq M$. It is called semiprime if for any nonempty subset $A$ of $S$ such that $A * A \subseteq M$, we have $A \subseteq M$.

Definition 2.11. [15] Let $(S, \circ)$ be an hypergroupoid or an ordered hypergroupoid. A subset $M$ of $S$ is called weakly prime if for any ideals $A, B$ of $S$ such that $A * B \subseteq M$, we have $A \subseteq M$ or $B \subseteq M$. It is called weakly semiprime if for any ideal $A$ of $S$ such that $A * A \subseteq M$, we have $A \subseteq M$.

For a nonempty subset $A$ of a semigroup, ordered semigroup, $\Gamma$-semigroup, ordered $\Gamma$ semigroup, hypersemigroup or ordered hypersemigroup $S$, we denote by $I(A)$ the ideal of $S$ generated by $A$, and we have $I(A)=A \cup S A \cup A S \cup S A S, I(A)=(A \cup S A \cup A S \cup S A S]$, $I(A)=A \cup S \Gamma A \cup A \Gamma S \cup S \Gamma A \Gamma S, I(A)=(A \cup S \Gamma A \cup A \Gamma S \cup S \Gamma A \Gamma S], I(A)=A \cup S * A \cup$ $A * S \cup S * A * S, I(A)=(A \cup S * A \cup A * S \cup S * A * S]$, respectively; where for a subset $B$ of $S$, the symbol $(B]$ denotes the subset of $S$ defined by $\{t \in S \mid t \leq b$ for some $b \in B\}$.

When is convenient and no confusion is possible, we identify the singleton $\{a\}$ by the element $a$.

\section{On $\vee e$-semigroups}

Let $(S, \cdot, \leq)$ be a poe-groupoid. An element $m$ of $S$ is called weakly prime if for any ideal elements $a, b$ of $S$ such that $a b \leq m$ we have $a \leq m$ or $b \leq m$. It is called weakly semiprime if for any ideal element $a$ of $S$ such that $a^{2} \leq m$ we have $a \leq m$. An element $m$ of a poe-groupoid $S$ is called prime if for any elements $a, b$ of $S$ such that $a b \leq m$ we have $a \leq m$ or $b \leq m$. It is called semiprime if for any element $a$ of $S$ such that $a^{2} \leq m$ we have $a \leq m$. For a $\vee$-semigroup $S$ and an element $a$ of $S$, we denote by $i(a)$ the ideal element of $S$ generated by $a$, and we have $i(a)=a \vee e a \vee a e \vee e a e$.

Proposition 3.1. Let $S$ be $a$ Ve-semigroup and $m$ be an ideal element of $S$. The following are equivalent:

(1) $m$ is weakly prime.

(2) If $a, b \in F_{r}$ such that $a b \leq m$, then $a \leq m$ or $b \leq m$.

(3) If $a, b \in F_{l}$ such that $a b \leq m$, then $a \leq m$ or $b \leq m$.

(4) If $a \in F_{r}$ and $b \in F_{l}$ such that $a b \leq m$, then $a \leq m$ or $b \leq m$.

Proof. $(1) \Longrightarrow(2)$. Let $a, b \in F_{r}$ such that $a b \leq m$. We consider the ideal elements $i(a)$ and $i(b)$ of $S$ generated by $a$ and $b$, respectively, and we have

$$
i(a) i(b)=(a \vee e a \vee a e \vee e a e)(b \vee e b \vee b e \vee e b e)
$$




$$
=a b \vee e a b \vee a e b \vee e a e b \vee a b e \vee \text { eabe } \vee \text { aebe } \vee \text { eaebe. }
$$

We have $a b \leq m, e(a b) \leq e m \leq m,(a e) b \leq a b \leq m, e(a e) b \leq e a b \leq m,(a b) e \leq m e \leq m$, $e(a b e) \leq e m \leq m,(a e)(b e) \leq a b \leq m, e(a e b e) \leq e m \leq m$. Thus we have $i(a) i(b) \leq m$. Since $m$ is weakly prime and $i(a), i(b)$ are ideal elements of $S$, we have $i(a) \leq m$ or $i(b) \leq m$, thus we have $a \leq m$ or $b \leq m$ and property (2) holds.

$(1) \Longrightarrow(3)$. Let $a, b \in F_{l}$ and $a b \leq m$. We have

$$
i(a) i(b)=a b \vee e a b \vee a e b \vee e a e b \vee a b e \vee \text { eabe } \vee \text { aebe } \vee \text { eaebe. }
$$

We also have $a b \leq m, e(a b) \leq e m \leq m, a(e b) \leq a b \leq m,(e a)(e b) \leq a b \leq m,(a b) e \leq$ $m e \leq m, e(a b e) \leq e m \leq m,(a e b) e \leq m e \leq m, e(a e b e) \leq e m \leq m$. Then $i(a) i(b) \leq m$ and, by (1), $i(a) \leq m$ or $i(b) \leq m$ and so $a \leq m$ or $b \leq m$.

$(1) \Longrightarrow(4)$. Let $a \in F_{r}, b \in F_{l}$ and $a b \leq m$. We have

$$
i(a) i(b)=a b \vee e a b \vee a e b \vee e a e b \vee \text { abe } \vee \text { eabe } \vee \text { aebe } \vee \text { eaebe, }
$$

$a b \leq m, e(a b) \leq e m \leq m,(a e) b \leq a b \leq m, e(a e b) \leq e m \leq m,(a b) e \leq m e \leq m$, $e(a b e) \leq e m \leq m, a(e b) e \leq a b e \leq m, e(a e b e) \leq e m \leq m$. By hypothesis, we have $i(a) \leq m$ or $i(b) \leq m$ and so $a \leq m$ or $b \leq m$.

The implications $(2) \Rightarrow(1),(3) \Rightarrow(1)$ and $(4) \Rightarrow(1)$ are obvious and they hold in po-groupoids in general.

Proposition 3.2. Let $S$ be $a \vee$ vesemigroup and $m$ an ideal element of $S$. The following are equivalent:

(1) $m$ is weakly semiprime.

(2) If $a \in F_{r}$ such that $a^{2} \leq m$, then $a \leq m$.

(3) If $b \in F_{l}$ such that $b^{2} \leq m$, then $b \leq m$.

Proof. $(1) \Longrightarrow(2)$. Let $a \in F_{r}$ and $a^{2} \leq m$. Then

$$
\begin{aligned}
i(a)^{2} & =(a \vee e a \vee a e \vee e a e)(a \vee e a \vee a e \vee e a e) \\
& =a^{2} \vee e a^{2} \vee a e a \vee e a e a \vee a^{2} e \vee e a^{2} e \vee a e a e \vee \text { eaeae. }
\end{aligned}
$$

We have $a^{2} \leq m, e a^{2} \leq e m \leq m,(a e) a \leq a^{2} \leq m, e($ aea $) \leq e m \leq m, a^{2} e \leq m e \leq m$, $e\left(a^{2} e\right) \leq e m \leq m,(a e)(a e) \leq a^{2} \leq m, e(a e a e) \leq e m \leq m$. Since $i(a)$ is an ideal element of $S$ and $i(a)^{2} \leq m$, by (1), we have $i(a) \leq m$ and so $a \leq m$.

$(1) \Longrightarrow(3)$. Let $b \in F_{l}$ and $b^{2} \leq m$. Then

$$
i(b)^{2}=b^{2} \vee e b^{2} \vee b e b \vee e b e b \vee b^{2} e \vee e b^{2} e \vee \text { bebe } \vee \text { ebebe. }
$$

We have $b^{2} \leq m, e b^{2} \leq e m \leq m, b(e b) \leq b^{2} \leq m,(e b)(e b) \leq b^{2} \leq m, b^{2} e \leq m e \leq m$, $e\left(b^{2} e\right) \leq e m \leq m,(b e b) e \leq m e \leq m, e(b e b e) \leq e m \leq m$. Since $i(b)$ is an ideal element of $S$ and $i(b)^{2} \leq m$, by (1), we have $i(b) \leq m$ and $b \leq m$.

The implications $(2) \Rightarrow(1)$ and $(3) \Rightarrow(1)$ are obvious and they hold in po-groupoids in general. 


\section{Applications to semigroups, $\Gamma$-semigroups and to hypersemigroups}

In this section, we apply the above results to semigroups, $\Gamma$-semigroups, and to hypersemigroups.

Let us begin with a semigroup. As an application of Proposition 3.1 to semigroups, we have the following corollary.

Corollary 4.1. Let $(S, \cdot)$ be a semigroup and $M$ an ideal of $S$. The following are equivalent:

(1) $M$ is weakly prime.

(2) If $A, B$ are right ideals of $S$ such that $A B \subseteq M$, then $A \subseteq M$ or $B \subseteq M$.

(3) If $A, B$ are left ideals of $S$ such that $A B \subseteq M$, then $A \subseteq M$ or $B \subseteq M$.

(4) If $A$ is a right ideal and $B$ is a left ideal of $S$ such that $A B \subseteq M$, then $A \subseteq M$ or $B \subseteq M$.

Proof. (1) $\Longrightarrow(2)$. Let $A, B$ be right ideals of $S$ such that $A B \subseteq M$. The set $\mathcal{P}(S)$ of all subsets of $S$ with the multiplication $A \circ B:=A B$ and the inclusion relation " $\subseteq$ " is a $\vee e$-semigroup, $M$ is a weakly prime ideal element of $(\mathcal{P}(S), \circ, \subseteq)$ and $A, B$ are right ideal elements of $(\mathcal{P}(S), \circ, \subseteq)$ such that $A \circ B \subseteq M$. By Proposition 3.1(1) $\Rightarrow(2)$, we have $A \subseteq M$ or $B \subseteq M$, so property (2) is satisfied. The implications (1) $\Rightarrow(3)$ and (1) $\Rightarrow(4)$ can be proved in a similar way. The implications $(2) \Rightarrow(1),(3) \Rightarrow(1)$ and $(4) \Rightarrow(1)$ are obvious and they hold in groupoids in general.

In a similar way, applying Proposition 3.2 to semigroups we get the following corollary.

Corollary 4.2. Let $S$ be a semigroup and $M$ an ideal of $S$. The following are equivalent:

(1) $M$ is weakly semiprime.

(2) If $A$ is a right ideal of $S$ such that $A^{2} \subseteq M$, then $A \subseteq M$.

(3) If $B$ is a left ideal of $S$ such that $B^{2} \subseteq M$, then $B \subseteq M$.

We apply now Proposition 3.1 to $\Gamma$-semigroups.

Corollary 4.3. Let $(S, \Gamma)$ be a $\Gamma$-semigroup and $M$ be an ideal of $S$. The following are equivalent:

(1) $M$ is weakly prime.

(2) If $A, B$ are right ideals of $S$ such that $A \Gamma B \subseteq M$, then $A \subseteq M$ or $B \subseteq M$.

(3) If $A, B$ are left ideals of $S$ such that $A \Gamma B \subseteq M$, then $A \subseteq M$ or $B \subseteq M$.

(4) If $A$ is a right ideal and $B$ is a left ideal of $S$ such that $A \Gamma B \subseteq M$, then $A \subseteq M$ or $B \subseteq M$. 
Proof. $(1) \Longrightarrow(2)$. Let $A, B$ be right ideals of $S$ such that $A \Gamma B \subseteq M$. The set $\mathcal{P}(S)$ of all subsets of $S$ with the multiplication $A \circ B=A \Gamma B$ and the inclusion relation "ᄃ" is a $\vee e$-semigroup, the set $M$ is a weakly prime ideal element of $(\mathcal{P}(S), \circ, \subseteq)$ and, $A, B$ are ideal elements of $(\mathcal{P}(S), \circ, \subseteq)$ such that $A \circ B \subseteq M$. By Proposition 3.1, we have $A \subseteq M$ or $B \subseteq M$.

In a similar way, Proposition 3.2 can be applied to the following corollary.

Corollary 4.4. Let $(S, \Gamma)$ be a $\Gamma$-semigroup and $M$ an ideal of $S$. The following are equivalent:

(1) $M$ is weakly semiprime.

(2) If $A$ is a right ideal of $S$ such that $A \Gamma A \subseteq M$, then $A \subseteq M$.

(3) If $B$ is a left ideal of $S$ such that $B \Gamma B \subseteq M$, then $B \subseteq M$.

Finally, we apply Proposition 3.1 to hypersemigroups.

Corollary 4.5. Let $(S, \circ)$ be an hypersemigroup and $M$ be an ideal of $S$. The following are equivalent:

(1) $M$ is weakly prime.

(2) If $A, B$ are right ideals of $S$ such that $A * B \subseteq M$, then $A \subseteq M$ or $B \subseteq M$.

(3) If $A, B$ are left ideals of $S$ such that $A * B \subseteq M$, then $A \subseteq M$ or $B \subseteq M$.

(4) If $A$ is a right ideal and $B$ a left ideal of $S$ such that $A * B \subseteq M$, then $A \subseteq M$ or $B \subseteq M$.

Proof. $(1) \Longrightarrow(2)$. Let $A, B$ be right ideals of $(S, \circ)$ such that $A * B \subseteq M$. By Lemma 2.5 , the set $\mathcal{P}^{*}(S)$ of all nonempty subsets of $S$ with the multiplication $A \bullet B:=A * B$ and the inclusion relation " $\subseteq$ " is a $\vee e$-semigroup; the set $M$ is a weakly prime ideal element of $\left(\mathcal{P}^{*}(S), \bullet, \subseteq\right)$ and $A, B$ are right ideal element of $\left(\mathcal{P}^{*}(S), \bullet, \subseteq\right)$ such that $A \bullet B \subseteq M$. By Proposition 3.1(1) $\Rightarrow(2)$, we have $A \subseteq M$ or $B \subseteq M$ and property (2) holds. The rest of the corollary can be proved at a similar way.

Similarly, applying Proposition 3.2 to hypersemigroups, we get the following corollary.

Corollary 4.6. Let $(S, \circ)$ be an hypersemigroup and $M$ an ideal of $S$. The following are equivalent:

(1) $M$ is weakly semiprime.

(2) If $A$ is a right ideal of $S$ such that $A * A \subseteq M$, then $A \subseteq M$.

(3) If $B$ is a left ideal of $S$ such that $B * B \subseteq M$, then $B \subseteq M$.

If we want to obtain the results of this section independently, then their proof is on the line of the corresponding proofs of the $\vee e$-semigroups given in the previous section. 


\section{On ordered semigroups}

If $S$ is an ordered semigroup then, for any nonempty subsets $A$ and $B$ of $S$, we have $(A](B] \subseteq(A B] ; A \subseteq B \Rightarrow(A] \subseteq(B] ;$ and if $M$ is an ideal of $S$, then $(M]=M$. Using these properties, we prove the following proposition. For the sake of completeness we will give its proof.

Proposition 5.1. (cf. also [7; the Theorem]) Let $(S, \cdot, \leq)$ be an ordered semigroup and $M$ be an ideal of $S$. The following are equivalent:

(1) $M$ is weakly prime.

(2) If $A, B$ are right ideals of $S$ such that $A B \subseteq M$, then $A \subseteq M$ or $B \subseteq M$.

(3) If $A, B$ are left ideals of $S$ such that $A B \subseteq M$, then $A \subseteq M$ or $B \subseteq M$.

(4) If $A$ is a right ideal and $B$ a left ideal of $S$ such that $A B \subseteq M$, then $A \subseteq M$ or $B \subseteq M$.

Proof. (1) $\Longrightarrow(2)$. Let $A, B$ be right ideals of $S$ such that $A B \subseteq M$. We consider the ideals $I(A)$ and $I(B)$ of $S$ generated by $A$ and $B$ respectively. We have

$$
\begin{aligned}
I(A) I(B) & =(A \cup S A \cup A S \cup S A S](B \cup S B \cup B S \cup S B S] \\
& \subseteq((A \cup S A \cup A S \cup S A S)(B \cup S B \cup B S \cup S B S)] \\
& =(A B \cup S A B \cup A S B \cup S A S B \cup A B S \cup S A B S \cup A S B S \cup S A S B S] \\
& \subseteq(M]=M .
\end{aligned}
$$

Since $I(A) I(B) \subseteq M$, by (1), we have $I(A) \subseteq M$ or $I(B) \subseteq M$ and so $A \subseteq M$ or $B \subseteq M$. $(1) \Longrightarrow(3)$. Let $A, B$ be left ideals of $S$ such that $A B \subseteq M$. In a similar way as in the previous case, we have $I(A) I(B) \subseteq M$, then $I(A) \subseteq M$ or $I(B) \subseteq M$ and so $A \subseteq M$ or $B \subseteq M$.

$(1) \Longrightarrow(4)$. Let $A$ be a right ideal and $B$ a left ideal of $S$ such that $A B \subseteq M$. Then again $I(A) I(B) \subseteq M$ and so $A \subseteq M$ or $B \subseteq M$.

The implications $(2) \Rightarrow(1),(3) \Rightarrow(1)$ and $(4) \Rightarrow(1)$ are obvious.

Although for an ordered semigroup $(S, \cdot, \leq)$, the set $\mathcal{P}(S)$ is again a $\vee e$-semigroup, one can easily check that Proposition 3.1 cannot be applied to ordered semigroups and an independent proof of Proposition 5.1 is needed; though its proof is again on the line of Proposition 3.1.

Proposition 5.2. (cf. also [7; Remark 4]) Let $S$ be an ordered semigroup and $M$ an ideal of $S$. The following are equivalent:

(1) $M$ is weakly semiprime.

(2) If $A$ is a right ideal of $S$ such that $A^{2} \subseteq M$, then $A \subseteq M$. 
(3) If $B$ is a left ideal of $S$ such that $B^{2} \subseteq M$, then $B \subseteq M$.

Note. The results on semigroups, that is Corollaries 4.1 and 4.2 can be also obtained as application of the corresponding results of this section. As an example, suppose $(S, \cdot)$ is a semigroup, $M$ a weakly prime ideal of $(S, \cdot)$ and $A, B$ be right ideals of $(S, \cdot)$ such that $A B \subseteq M$. We endow $(S, \cdot)$ with the order $\leq=\{(x, y) \mid x=y\}$. Then $(S, \cdot, \leq)$ is an ordered semigroup, the set $M$ is a weakly prime ideal of $(S, \cdot, \leq)$ and $A, B$ are ideals of $(S, \cdot, \leq)$ such that $A B \subseteq M$. By Proposition 5.1(1) $\Rightarrow(2)$, we have $A \subseteq M$ or $B \subseteq M$ and property (2) of Corollary 4.1 is satisfied (see also [7,8]; the introduction).

\section{On ordered $\Gamma$-semigroups}

For an ordered $\Gamma$-semigroup $S$ and nonempty subsets $A$ and $B$ of $S$, we have $(A] \Gamma(B] \subseteq$ $(A \Gamma B] ; A \subseteq B \Rightarrow(A] \subseteq(B]$; and if $M$ is an ideal of $S$, then $(M]=M$. Using these properties, we prove the following proposition.

Proposition 6.1. Let $S$ be an ordered $\Gamma$-semigroup and $M$ be an ideal of $S$. The following are equivalent:

(1) $M$ is weakly prime.

(2) If $A, B$ are right ideals of $S$ such that $A \Gamma B \subseteq M$, then $A \subseteq M$ or $B \subseteq M$.

(3) If $A, B$ are left ideals of $S$ such that $A \Gamma B \subseteq M$, then $A \subseteq M$ or $B \subseteq M$.

(4) If $A$ is a right ideal and $B$ a left ideal of $S$ such that $A \Gamma B \subseteq M$, then $A \subseteq M$ or $B \subseteq M$.

Proof. Let us prove the implication $(1) \Rightarrow(3)$. Let $A, B$ be left ideals of $S$ such that $A \Gamma B \subseteq M$. Then we have

$$
\begin{aligned}
I(A) \Gamma I(B)= & (A \cup S \Gamma A \cup A \Gamma S \cup S \Gamma A \Gamma S](B \cup S \Gamma B \cup B \Gamma S \cup S \Gamma B \Gamma S] \\
\subseteq & ((A \cup S \Gamma A \cup A \Gamma S \cup S \Gamma A \Gamma S)(B \cup S \Gamma B \cup B \Gamma S \cup S \Gamma B \Gamma S)] \\
= & (A \Gamma B \cup S \Gamma A \Gamma B \cup A \Gamma S \Gamma B \cup S \Gamma A \Gamma S \Gamma B \cup A \Gamma B \Gamma S \\
& \cup S \Gamma A \Gamma B \Gamma S \cup A \Gamma S \Gamma B \Gamma S \cup S \Gamma A \Gamma S \Gamma B \Gamma S] \\
\subseteq & (M]=M .
\end{aligned}
$$

Since $I(A) \Gamma I(B) \subseteq M$, by hypothesis, we have $I(A) \subseteq M$ or $I(B) \subseteq M$ and so $A \subseteq M$ or $B \subseteq M$.

The implications $(1) \Rightarrow(2)$ and $(1) \Rightarrow(4)$ can be proved at a similar way; and the implications $(2) \Rightarrow(1),(3) \Rightarrow(1)$ and $(4) \Rightarrow(1)$ are obvious.

In a similar way we prove the following proposition.

Proposition 6.2. Let $S$ be an ordered $\Gamma$-semigroup and $M$ an ideal of $S$. The following are equivalent: 
(1) $M$ is weakly semiprime.

(2) If $A$ is a right ideal of $S$ such that $A \Gamma A \subseteq M$, then $A \subseteq M$.

(3) If $B$ is a left ideal of $S$ such that $B \Gamma B \subseteq M$, then $B \subseteq M$.

The results on $\Gamma$-semigroups, that is Corollaries 4.3 and 4.4 can be also obtained as application of the corresponding results of this section in the way indicated in the Note of section 5 .

\section{On ordered hypersemigroups}

For an ordered hypersemigroup $(S, \circ, \leq)$ and nonempty subsets $A$ and $B$ of $S$, we have $(A] *(B] \subseteq(A * B][11] ; A \subseteq B \Rightarrow(A] \subseteq(B] ;$ and if $M$ is an ideal of $S$, then $(M]=M$. Using these properties, we prove the following proposition.

Proposition 7.1. Let $(S, \circ, \leq)$ be an ordered hypersemigroup and $M$ an ideal of $S$. The following are equivalent:

(1) $M$ is weakly prime.

(2) If $A, B$ are right ideals of $S$ such that $A * B \subseteq M$, then $A \subseteq M$ or $B \subseteq M$.

(3) If $A, B$ are left ideals of $S$ such that $A * B \subseteq M$, then $A \subseteq M$ or $B \subseteq M$.

(4) If $A$ is a right ideal and $B$ a left ideal of $S$ such that $A * B \subseteq M$, then $A \subseteq M$ or $B \subseteq M$.

Proof. Let us prove the implication $(1) \Rightarrow(4)$. Let $A$ be a right ideal and $B$ a left ideal of $S$ such that $A * B \subseteq M$. We have

$$
\begin{aligned}
I(A) * I(B)= & (A \cup S * A \cup A * S \cup S * A * S] *(B \cup S * B \cup B * S \cup S * B * S] \\
\subseteq & ((A \cup S * A \cup A * S \cup S * A * S)(B \cup S * B \cup B * S \cup S * B * S)] \\
= & (A * B \cup S * A * B \cup A * S * B \cup S * A * S * B \cup A * B * S \\
& \cup S * A * B * S \cup A * S * B * S \cup S * A * S * B * S] \\
\subseteq & (M]=M .
\end{aligned}
$$

Since $I(A) * I(B) \subseteq M$, by (1), we have $I(A) \subseteq M$ or $I(B) \subseteq M$ and so $A \subseteq M$ or $B \subseteq M$. The implications $(1) \Rightarrow(2)$ and $(1) \Rightarrow(3)$ can be proved at a similar way and the implications $(2) \Rightarrow(1),(3) \Rightarrow(1)$ and $(4) \Rightarrow(1)$ are obvious.

The proof of the following proposition is analogous.

Proposition 7.2. Let $(S, \circ, \leq)$ be an ordered hypersemigroup and $M$ an ideal of $S$. The following are equivalent:

(1) $M$ is weakly semiprime. 
(2) If $A$ is a right ideal of $S$ such that $A * A \subseteq M$, then $A \subseteq M$.

(3) If $B$ is a left ideal of $S$ such that $B * B \subseteq M$, then $B \subseteq M$.

The following note is referred to sections $5-7$.

Note. To pass from ordered semigroups to ordered $\Gamma$-semigroups or to ordered hypersemigroups, it is enough to put a " $\Gamma$ " or " $*$ " in the appropriate place.

\section{Another characterization of weakly prime ideal elements-ideals}

Proposition 8.1. Let $S$ be a poe-semigroup and $m \in S$. Suppose that for every right ideal element $a$ of $S$ and any $b \in S$, aeb $\leq m$ implies $a \leq m$ or $b \leq m$. Then $m$ is weakly prime.

Proof. Let $a, b$ be ideal elements of $S$ such that $a b \leq m$. Since $a$ is a right ideal element of $S, b \in S$ and $a e b=(a e) b \leq a b \leq m$, by hypothesis, we have $a \leq m$ or $b \leq m$, thus $m$ is weakly prime.

Proposition 8.2. Let $S$ be a poe-semigroup and $m \in S$. Suppose that for every left ideal element $b$ of $S$ and any $a \in S$, aeb $\leq m$ implies $a \leq m$ or $b \leq m$. Then $m$ is weakly prime.

Proof. Let $a, b$ be ideal elements of $S$ such that $a b \leq m$. Since $b$ is a left ideal element of $S, a \in S$ and $a e b=a(e b) \leq a b \leq m$, by hypothesis, we have $a \leq m$ or $b \leq m$, thus $m$ is weakly prime.

Corollary 8.3. Let $(S, \cdot)$ be a semigroup and $M$ a subset of $S$. Suppose that for any right ideal $A$ of $S$ and any $B \subseteq S$ (or for any left ideal $B$ of $S$ and any $A \subseteq S$ ), $A S B \subseteq M$, implies $A \subseteq M$ or $B \subseteq M$. Then $M$ is weakly prime.

Corollary 8.4. Let $(S, \Gamma)$ be a $\Gamma$-semigroup and $M$ a subset of $S$. Suppose that for any right ideal $A$ of $S$ and any $B \subseteq S$ (or for any left ideal $B$ of $S$ and any $A \subseteq S$ ), $A \Gamma S \Gamma B \subseteq M$, implies $A \subseteq M$ or $B \subseteq M$. Then $M$ is weakly prime.

For its proof, it is enough to remark that $(\mathcal{P}(S), \cdot, \subseteq)$ is a poe-semigroup $(\mathcal{P}(S)$ is the set of subsets of $S$ ). If we put $\Gamma=\{\cdot\}$, then Corollary 8.3 can be obtained.

Corollary 8.5. Let $(S, \circ)$ be an hypersemigroup and $M$ a subset of $S$. Suppose that for any right ideal $A$ of $S$ and any $B \subseteq S$ (or for any left ideal $B$ of $S$ and any $A \subseteq S$ ), $A * S * B \subseteq M$, implies $A \subseteq M$ or $B \subseteq M$. Then $M$ is weakly prime.

Proposition 8.6. Let $S$ be $a$ Ve-semigroup and $m$ an ideal element of $S$. If $m$ is weakly prime then, for every $a, b \in S$ such that aeb $\leq m$, we have $a \leq m$ or $b \leq m$.

Proof. Let $a, b \in S$ such that $a e b \leq m$. Then we have $(e a e)(e b e) \leq e(a e b) e \leq e m e \leq m$. Since eae, ebe are ideal elements of $S$ and $m$ is weakly prime, we have eae $\leq m$ or $e b e \leq m$. Let eae $\leq m$. Then

$$
\begin{aligned}
i(a)^{2} i(a) & =(a \vee e a \vee a e \vee e a e)^{2}(a \vee e a \vee a e \vee e a e) \\
& \leq(e a \vee e a e)(a \vee e a \vee a e \vee e a e) \\
& \leq e a e \leq m
\end{aligned}
$$


Since the elements $i(a)^{2}$ and $i(a)$ are ideal elements of $S$ and $m$ is weakly prime, we have $i(a)^{2} \leq m$ or $i(a) \leq m$. If $i(a) \leq m$, then we have $a \leq i(a) \leq m$. If $i(a)^{2} \leq m$ then, since $i(a)$ is an ideal element of $S$ and $m$ is weakly prime, we have $i(a) \leq m$ and so $a \leq m$. From ebe $\leq m$, by symmetry, we have $b \leq m$.

Corollary 8.7. Let $(S, \cdot)$ be a semigroup and $M$ an ideal of $S$. If $M$ is weakly prime then, for every subsets $A, B$ of $S$ such that $A S B \subseteq M$, we have $A \subseteq M$ or $B \subseteq M$.

Corollary 8.8. Let $(S, \Gamma)$ be a $\Gamma$-semigroup and $M$ an ideal of $S$. If $M$ is weakly prime then, for every subsets $A, B$ of $S$ such that $A \Gamma S \Gamma B \subseteq M$, we have $A \subseteq M$ or $B \subseteq M$.

Corollary 8.9. Let $(S, \circ)$ be an hypersemigroup and $M$ an ideal of $S$. If $M$ is weakly prime then, for every nonempty subsets $A, B$ of $S$ such that $A * S * B \subseteq M$, we have $A \subseteq M$ or $B \subseteq M$.

In addition, by Proposition 8.1 or Proposition 8.2 and Proposition 8.6, the following proposition holds.

Proposition 8.10. Let $S$ be $a \vee$ Ve-semigroup and $m$ an ideal element of $S$. The following are equivalent:

(1) $m$ is weakly prime.

(2) For every $a, b \in S$ such that $a e b \leq m$, we have $a \leq m$ or $b \leq m$.

Clearly, the analogous for semigroups, $\Gamma$-semigroups and hypersemigroups also holds. An ideal $M$ of an hypersemigroup $(S, \circ)$, for example is weakly prime if and only if for any nonempty subsets $A, B$ of $S$ such that $A * S * B \subseteq M$, we have $A \subseteq M$ or $B \subseteq M$. This is equivalent to saying that for any $a, b \in S$ such that $a * S * b \subseteq M$ we have $a \in M$ or $b \in M$. In the last characterization, if we delete the operation "*" and put "." instead, then this is the definition of a weakly prime ideal of a semigroup given by Petrich in $[17$; II.3.1 Definition]. Petrich uses the terms "prime", "completely prime" while we use the terms "weakly prime", "prime".

In the rest of this section, we consider the case of ordered semigroups, ordered $\Gamma$ semigroups and ordered hypersemigroups.

Proposition 8.11. Let $S$ be an ordered semigroup and $M$ be a subset of $S$ such that $M=(M]$. Suppose that for any right ideal $A$ of $S$ and any $B \subseteq S,(A S B] \subseteq M$ implies $A \subseteq M$ or $B \subseteq M$. Then $M$ is weakly prime.

Proof. Let $A, B$ be ideals of $S$ such that $A B \subseteq M$. Then $(A S B] \subseteq(A B] \subseteq(M]=M$. Since $A$ is a right ideal of $S, B \subseteq S$ and $(A S B] \subseteq M$, by hypothesis, we have $A \subseteq M$ or $B \subseteq M$, thus $M$ is weakly prime.

In a similar way the following proposition holds.

Proposition 8.12. Let $S$ be an ordered semigroup and $M$ be a subset of $S$ such that $M=(M]$. Suppose that for any left ideal $B$ of $S$ and any $A \subseteq S,(A S B] \subseteq M$ implies $A \subseteq M$ or $B \subseteq M$. Then $M$ is weakly prime. 
Proposition 8.13. Let $S$ be an ordered semigroup and $M$ be an ideal of $S$. Suppose that for any right ideal $A$ of $S$ and any $B \subseteq S, A S B \subseteq M$ implies $A \subseteq M$ or $B \subseteq M$. Then $M$ is weakly prime.

Proposition 8.14. Let $S$ be an ordered semigroup and $M$ be an ideal of $S$. Suppose that for any left ideal $B$ of $S$ and any $A \subseteq S, A S B \subseteq M$ implies $A \subseteq M$ or $B \subseteq M$. Then $M$ is weakly prime.

The proof of the last two propositions mimics the proof of Propositions 8.11 and 8.12; however they can be also obtained as corollaries to Propositions 8.11 and 8.12.

Proposition 8.15. Let $S$ be an ordered semigroup and $M$ an ideal of $S$. If $M$ is weakly prime then, for any $A, B \subseteq S$ such that $(A S B] \subseteq M$, we have $A \subseteq M$ or $B \subseteq M$.

Proof. For $A=\emptyset$ or $B=\emptyset$, the proposition holds. Let $A, B$ nonempty subsets of $S$ such that $(A S B] \subseteq M$. Then we have

$$
\begin{aligned}
(S A S](S B S] & \subseteq\left(S A S^{2} B S\right] \subseteq(S(A S B) S]=(S(A S B] S] \subseteq(S M S] \\
& \subseteq(M]=M .
\end{aligned}
$$

Since $(S A S],(S B S]$ are ideals of $S$ and $M$ is weakly prime, we have $(S A S] \subseteq M$ or $(S B S] \subseteq M$. Let $(S A S] \subseteq M$. Then

$$
\begin{aligned}
I(A)^{3} & =(A \cup S A \cup A S \cup S A S]^{2}(A \cup S A \cup A S \cup S A S] \\
& \subseteq(S A \cup S A S](A \cup S A \cup A S \cup S A S] \\
& \subseteq((S A \cup S A S)(A \cup S A \cup A S \cup S A S)] \\
& \subseteq(S A S] \subseteq M .
\end{aligned}
$$

Then we have

$$
\left(I(A)^{2}\right] I(A)=\left(I(A)^{2}\right](I(A)] \subseteq\left(I(A)^{3}\right] \subseteq(M]=M .
$$

Since $I(A)$ is an ideal of $S,\left(I(A)^{2}\right]$ is an ideal of $S$ as well (in general, $A, B$ ideals $\Rightarrow(A B]$ ideal). Since $\left(I(A)^{2}\right], I(A)$ are ideals of $S$ and $M$ is weakly prime, we have $\left(I(A)^{2}\right] \subseteq M$ or $I(A) \subseteq M$. If $I(A) \subseteq M$, then $A \subseteq M$. If $\left(I(A)^{2}\right] \subseteq M$, then $I(A)^{2} \subseteq\left(I(A)^{2}\right] \subseteq M$. Since $I(A)$ is an ideal of $S$ and $M$ is weakly prime, we have $I(A) \subseteq M$ and again $A \subseteq M$.

Remark 8.16. If $S$ is an ordered semigroup and $M$ is an ideal of $S$, then the following are equivalent:

(1) for any $A, B \subseteq S,(A S B] \subseteq M$ implies $A \subseteq M$ or $B \subseteq M$.

(2) for any $A, B \subseteq S, A S B \subseteq M$ implies $A \subseteq M$ or $B \subseteq M$.

By Proposition 8.11 (or 8.12), Proposition 8.15 and Remark 8.16, the following proposition holds.

Proposition 8.17. (see also [7; the Theorem]) Let $(S, \cdot)$ be an ordered semigroup and $M$ an ideal of $S$. The following are equivalent: 
(1) $M$ is weakly prime.

(2) For any subsets $A$ and $B$ of $S$ such that $(A S B] \subseteq M$, we have $A \subseteq M$ or $B \subseteq M$.

(3) For any $a, b \in S$ such that $(a S b] \subseteq M$, we have $a \in M$ or $b \in M$.

(4) For any subsets $A$ and $B$ of $S$ such that $A S B \subseteq M$, we have $A \subseteq M$ or $B \subseteq M$.

(5) For any $a, b \in S$ such that $a S b \subseteq M$, we have $a \in M$ or $b \in M$.

The above results on ordered semigroups can be transferred to ordered $\Gamma$-semigroups (resp. ordered hypersemigroups) by putting a " $\Gamma$ " (resp. "*") in the appropriate place and we have the following results.

Proposition 8.18. Let $(S, \cdot)$ be an ordered $\Gamma$-semigroup and $M$ an ideal of $S$. The following are equivalent:

(1) $M$ is weakly prime.

(2) For any subsets $A$ and $B$ of $S$ such that $(A \Gamma S \Gamma B] \subseteq M$, we have $A \subseteq M$ or $B \subseteq M$.

(3) For any $a, b \in S$ such that $(a \Gamma S \Gamma b] \subseteq M$, we have $a \in M$ or $b \in M$.

(4) For any subsets $A$ and $B$ of $S$ such that $A \Gamma S \Gamma B \subseteq M$, we have $A \subseteq M$ or $B \subseteq M$.

(5) For any $a, b \in S$ such that $a \Gamma S \Gamma b \subseteq M$, we have $a \in M$ or $b \in M$.

Proposition 8.19. Let $(S, \circ)$ be an ordered hypersemigroup and $M$ an ideal of $S$. The following are equivalent:

(1) $M$ is weakly prime.

(2) For every nonempty subsets $A$ and $B$ of $S$ such that $(A * S * B] \subseteq M$, we have $A \subseteq M$ or $B \subseteq M$.

(3) For every $a, b \in S$ such that $(a * S * b] \subseteq M$, we have $a \in M$ or $b \in M$.

(4) For every nonempty subsets $A$ and $B$ of $S$ such that $A * S * B \subseteq M$, we have $A \subseteq M$ or $B \subseteq M$.

(5) For every $a, b \in S$ such that $a * S * b \subseteq M$, we have $a \in M$ or $b \in M$.

By easy modification of the proof of Proposition 8.17, the following proposition holds.

Proposition 8.20. (see also [7; Remark 4]) For an ideal $M$ of an ordered semigroup $S$, the following are equivalent:

(1) $M$ is weakly semiprime.

(2) For any subset $A$ of $S$ such that $(A S A] \subseteq M$, we have $A \subseteq M$. 
(3) For any $a \in S$ such that $(a S a] \subseteq M$, we have $a \in M$.

(4) For any subset $A$ of $S$ such that $A S A \subseteq M$, we have $A \subseteq M$.

(5) For any $a \in S$ such that $a S a \subseteq M$, we have $a \in M$.

The analogous result for ordered $\Gamma$-semigroups and ordered hypersemigroups also holds. In case of an ordered hypersemigroup $S$, the subset $A$ of $S$ in properties (2) and (4) of the above proposition should be a nonempty set.

\section{Some further results related to ordered hypergroupoids}

Let $(S, \cdot, \leq)$ be an ordered groupoid and "o" the hyperoperation on $S$ defined by

$$
a \circ b:=\{x \in S \mid x \leq a b\}
$$

and the same order " $\leq$ ". Then $(S, \circ, \leq)$ is an ordered hypergroupoid. In particular, if $(S, \cdot, \leq)$ is an ordered semigroup, then $(S, \circ, \leq)$ is an ordered hypersemigroup [16; Lemma 1].

Proposition 9.1. We have $A * B=(A B]$.

Proof. Indeed, we have

$$
\begin{aligned}
x \in A * B & \Longleftrightarrow x \in a \circ b \text { for some } a \in A, b \in B \\
& \Longleftrightarrow x \leq a b \in A B \\
& \Longleftrightarrow x \in(A B] .
\end{aligned}
$$

Proposition 9.2. If $M$ is a prime (resp. semiprime) subset of an ordered groupoid $(S, \cdot, \leq)$, then it is a prime (resp. semiprime) subset of the ordered hypergroupoid $(S, \circ, \leq)$. Proof. Let $M$ be a prime subset of $(S, \cdot, \leq)$ and $A, B$ be nonempty subsets of $(S, \circ, \leq)$ such that $A * B \subseteq M$. By Proposition 9.1, we have $A B \subseteq(A B]=A * B \subseteq M$. By hypothesis, we have $A \subseteq M$ or $B \subseteq M$, thus $M$ is a prime subset of $(S, \circ, \leq)$. Similarly, if $M$ is a semiprime subset of $(S, \cdot, \leq)$ and $A$ is a nonempty subset of $S$ such that $A * A \subseteq M$ then, by Proposition 9.1, we have $A^{2} \subseteq\left(A^{2}\right] \subseteq A * A \subseteq M$ and so $A \subseteq M$, thus $M$ is a semiprime subset of $(S, \circ, \leq)$.

Proposition 9.3. [16; Theorem 3] $A$ set $M$ is an ideal of an ordered groupoid $(S, \cdot, \leq)$ if and only if $M$ is an ideal of the ordered hypergroupoid $(S, \circ, \leq)$.

Proposition 9.4. A set $M$ is a prime (resp. semiprime) ideal of an ordered groupoid $(S, \cdot, \leq)$ if and only if $M$ is a prime ideal of the ordered hypergroupoid $(S, \circ, \leq)$.

Proof. $\Longrightarrow$. This follows from Propositions 9.2 and 9.3.

$\Longleftarrow$. Let $M$ be a prime ideal of $(S, \circ, \leq)$ and $A, B$ be subsets of $(S, \cdot, \leq)$ such that $A B \subseteq M$. By Proposition 9.1, we have $A * B=(A B] \subseteq(M]=M$. By hypothesis, we have $A \subseteq M$ 
or $B \subseteq M$, thus $M$ is a prime ideal of $(S, \cdot, \leq)$. The proof for semiprime ideals is similar.

Proposition 9.4 remains true if we replace the word "prime" by "weakly prime" and the following proposition holds.

Proposition 9.5. A set $M$ is a weakly prime ideal of an ordered groupoid $(S, \cdot, \leq)$ if and only if $M$ is a weakly prime ideal of the ordered hypergroupoid $(S, \circ, \leq)$.

I would like to thank the anonymous referee for his/her time to read the paper carefully and his/her prompt reply.

\section{References}

[1] G. Birkhoff. Lattice theory. Revised reprint of the 1948 edition. American Mathematical Society Colloquium Publications, 25. American Mathematical Society, Providence, R.I. $1960 \mathrm{xiii}+283 \mathrm{pp}$.

[2] G. Birkhoff. Lattice theory. Corrected reprint of the 1967 third edition. American Mathematical Society Colloquium Publications, 25. American Mathematical Society, Providence, R.I. 1979 vi+418 pp.

[3] A.H. Clifford, G.B. Preston. The algebraic theory of semigroups. Vol. I. Mathematical Surveys, No. 7 American Mathematical Society, Providence, R.I. 1961 xv+224 pp.

[4] L. Fuchs. Partially ordered algebraic systems. Pergamon Press, Oxford-London-New York-Paris; Addison-Wesley Publishing Co., Inc., Reading, Mass.-Palo Alto, Calif.London $1963 \mathrm{ix}+229 \mathrm{pp}$.

[5] Y. Hirano, E. Poon, H. Tsutsui. On rings in which every ideal is weakly prime. Bull. Korean Math. Soc. 47(5):1077-1087, 2010.

[6] N. Kehayopulu. On intra-regular Ve-semigroups. Semigroup Forum 19(2):111-121, 1980.

[7] N. Kehayopulu. On weakly prime ideals of ordered semigroups. Math. Japon. 35(6):1051-1056, 1990.

[8] N. Kehayopulu. On prime, weakly prime ideals in ordered semigroups. Semigroup Forum 44(3):341-346, 1992.

[9] N. Kehayopulu. On prime, weakly prime ideals in po-Г-semigroups. Lobachevskii J. Math. 30(4):257-262, 2009.

[10] N. Kehayopulu. On ordered Г-semigroups. Sci. Math. Jpn. 71(2):179-185, 2010.

[11] N. Kehayopulu. On hypersemigroups. Pure Math. Appl. (PU.M.A.) 25(2):151-156, 2015. 
[12] N. Kehayopulu. Left regular and intra-regular ordered hypersemigroups in terms of semiprime and fuzzy semiprime subsets. Sci. Math. Jpn. 80(3):295-305, 2017.

[13] N. Kehayopulu. How we pass from semigroups to hypersemigroups. Lobachevskii J. Math. 39(1):121-128, 2018.

[14] N. Kehayopulu. An application of $\Gamma$-semigroups techniques to the Green's theorem. Afr. Mat. 29(1-2):65-71, 2018.

[15] N. Kehayopulu. On ordered hypersemigroups with idempotent ideals, prime or weakly prime ideals. Eur. J. Pure Appl. Math. 11(1):10-22, 2018.

[16] N. Kehayopulu. On ordered hypersemigroups given by a table of multiplication and a figure. Turkish J. Math. 42(4):2045-2060, 2018.

[17] M. Petrich. Introduction to semigroups. Merrill Research and Lecture Series. Charles E. Merrill Publishing Co., Columbus, Ohio 1973 viii+198 pp.

[18] B. Pibaljommee, K. Wannatong, B. Davvaz. An investigation on fuzzy hyperideals of ordered semihypergroups. Quasigroups Related Systems 23(2):297-308, 2015.

[19] M.K. Sen, N.K. Saha. On Г-semigroup. I. Bull. Calcutta Math. Soc. 78(3):180-186, 1986.

[20] M.K. Sen, A. Seth. On po-Г-semigroups. Bull. Calcutta Math. Soc. 85(5):445-450, 1993. 